\title{
Tuberculosis in alpaca (Lama pacos) on a farm in Ireland. 1. A clinical report
}

\author{
Ryan, E.G. ${ }^{1}$, Dwyer, P.J. ${ }^{2}$, Connolly, D.J. ${ }^{3}$, Fagan, J. ${ }^{2}$, Costello, E. ${ }^{4}$ and More, S.J..$^{1,5}$ \\ ${ }^{1}$ Section of Herd and Veterinary Public Health, UCD School of Agriculture, Food Science and Veterinary \\ Medicine, University College Dublin, Belfield, Dublin 4, Ireland \\ ${ }^{2}$ Department of Agriculture, Fisheries and Food, Regional Veterinary Laboratory, Coosan, Athlone, Co. \\ Westmeath, Ireland \\ ${ }^{3}$ Ballybaun, Gort, Co. Galway, Ireland \\ ${ }^{4}$ Department of Agriculture, Fisheries and Food, Central Veterinary Research Laboratory, Backweston \\ Campus, Celbridge, Co. Kildare, Ireland \\ ${ }^{5}$ Centre for Veterinary Epidemiology and Risk Analysis, UCD School of Agriculture, Food Science and \\ Veterinary Medicine, University College Dublin, Belfield, Dublin 4, Ireland
}

\begin{abstract}
This case report describes tuberculosis (TB) due to infection with Mycobacterium bovis (M. bovis) in alpaca (Lama pacos) on a farm in Ireland. Two severely debilitated alpaca were presented to the University Veterinary Hospital, University College Dublin in November 2004. Bloods were taken, and haematology and biochemistry results were indicative of chronic infection. Radiological examination showed evidence of diffuse granulomatous pneumonia suggestive of tuberculosis. On necropsy there were granulomatous lesions present throughout many body organs including lung, liver, kidney, intestine as well on peritoneum and mesentery. Culture of acid-fast bacilli from lesions led to a diagnosis of tuberculosis due to M. bovis. The use of intradermal skin testing proved inefficient and unreliable for ante mortem diagnosis of tuberculosis in alpaca. Infection due to $M$. bovis should be considered among the differential diagnoses of debilitating diseases in alpaca, particularly those farmed in areas known to be traditional black spots for tuberculosis in cattle.
\end{abstract}

Key Words: albumin, alpaca, bovine tuberculosis, clinical examination, diagnosis, granulomatous pneumonia, intradermal skin testing, Lama pacos, lymphocyte proliferation assay, Mycobacterium bovis, necropsy, radiological examination

\section{Corresponding author:}

Eoin Ryan

Mulclair Veterinary Clinic,

Cappamore,

Co. Limerick and Newport, Co. Tipperary, Ireland

$\underline{\text { Irish Veterinary Journal }}$

Tel: +35387 7970478

\section{Introduction}

This case report describes tuberculosis (TB) due to infection with Mycobacterium bovis in alpaca (Lama pacos) on a farm in Ireland.

The alpaca herd was established in Ireland following the importation of 16 foundation stock ( 15 adults and one juvenile male) from Peru and Chile in 2000, nine from Northern Ireland in 2002 and four from Australia in 2003. The imported animals had all passed through quarantine (which included tuberculin testing using the single intradermal comparative cervical test, SICCT) in Belgium or Northern Ireland, en route to Ireland.They were farmed on newly reclaimed, double-fenced land, contiguous to cattle herds, in an area populated by badgers and wild goats. An eclectic range of health problems was noted from the outset, resulting in some deaths, including: skin disease, strongylosis, weight loss, fascioliasis, rickets, cardiomyopathy, trauma and 'chill'. Some alpacas died unexpectedly. The local veterinary practitioner was requested to investigate some of these deaths. However, few losses were examined post mortem, mainly due to legislative and commercial factors inhibiting on-farm post mortem examination and also due to distance of the alpaca farm from diagnostic laboratories. In October 2004, following the earlier death of three alpaca (A, B and C), the owner sought assistance with three ill alpaca.

\section{Investigation: on-farm and ARVL}

The farm was visited on November 8, 2004 by the local veterinary practitioner and a veterinary research officer from the Athlone Regional Veterinary Laboratory (ARVL). The disease history and health measures of the herd were reviewed. Contiguous herd history was obtained (Connolly et al., 2008), and clinical evaluation was carried out on the three ill animals. During the visit, it was noted that all three alpacas, two adults $\mathrm{D}$ and $\mathrm{E}$ and a juvenile of about six months old showed low body condition score of 1.5-2 (using a condition score range between 0 and 5, Australian Alpaca Association Inc., 2001) and poor appetite. Additionally, alpaca D showed severe dehydration, listlessness, a subnormal rectal temperature $\left(36.7^{\circ} \mathrm{C}\right.$; normal range is $37.5-38.9^{\circ} \mathrm{C}$ ) and shallow breathing with a mild expiratory grunt. Harsh lung sounds were audible on auscultation, but no palpable enlargement of surface lymph nodes was

Table 1: Key clinical and clinicopathological findings associated with TB in alpaca during this investigation

\begin{tabular}{|c|c|c|}
\hline Clinical findings & Haematology findings & Biochemistry findings \\
\hline Severe weight loss & Anaemia & Hypoalbuminaemia \\
\hline Harsh lung sounds & Leucocytosis & Hyperglobulinaemia \\
\hline Chronic ill thrift & Neutrophilia & $\begin{array}{l}\text { Albumin:globulin ratio } \\
\text { less than one }\end{array}$ \\
\hline
\end{tabular}


evident. Faecal samples were collected from 11 animals (including the three sick alpacas) for: Ziehl-Nielsen (ZN) staining for acid-fast bacilli; culturing for Salmonella; and parasitology. Blood samples were taken from six animals for clinical pathology. Testing was conducted at ARVL. A summary of the key clinical and clinicopathological findings is presented in Table 1. Briefly, all faecal samples were negative for Salmonella spp. on culture and acid-fast bacilli on ZN staining, and no nematode or fluke eggs were seen. Blood selenium values from the six animals ranged from 21-101 (average 38) glutathione peroxidise (GSH-Px) ovine units/ml. A further assessment of on-farm selenium status was conducted three weeks later (22 alpaca, range 12-82 [average 30] GSH-Px ovine units/ml) providing further evidence of a low to marginal selenium status. Blood copper values from the initial six animals were normal. In the three symptomatic animals, there was a raised globulin value with depressed albumin, with an albumin to globulin (A:G) ratio of 0.22 (for D), 0.47 and 0.59 . The mean A:G ratio for these three animals was 0.42 , whereas the clinically normal animals yielded a mean A:G ratio of 1.4. Tuberculin testing was conducted on the three ill animals using the SICCT test in the skin of the neck or by using the single intradermal comparative axillary tuberculin (SICAT) test in the skin of the axilla. In each case, insulin needles were used to ensure intradermal injection of $0.1 \mathrm{ml}$ of standard avian and bovine purified protein derivative (PPD). All three animals showed negative responses when the test was read 72 hours later. Nonetheless, given the clinical features, including weight loss and respiratory signs, clinical TB was suspected in these animals, supported by the presence of TB in local badgers and contiguous cattle herds.

\section{Investigation: University Veterinary Hospital General}

On November 17, 2004, animals D and E, and a further two healthy cohort alpacas were sent for examination to the University Veterinary Hospital (UVH), University College Dublin, including clinical examination, radiology, and clinical pathology. No further action was taken with the six-month old juvenile, which had recovered uneventfully. Clinically, D was found to be dull, weak, dehydrated, severely emaciated and had pale mucous membranes. Harsh lung sounds were diffusely evident on auscultation. Alpaca E was brighter and stronger, but was also severely emaciated, and harsh lung sounds were noted on auscultation. Animal D was anaemic and had a marked leucocytosis, neutrophilia and lymphocytosis. 'Low albumin: high globulin' was extremely marked, with an A:G of 0.53. Alpaca E was also anaemic; however, there was no evidence of a leucocytosis. Low albumin and high globulin levels were also marked, with an A:G of 0.59 . On radiography (Figure 1a and $\mathbf{1 b}$ ), D showed severe, diffuse consolidation of the lung fields consistent with granulomatous, broncho-interstitial pneumonia. Patchy consolidation was observed in lung radiographs from $\mathrm{E}$. In each case, TB was strongly suspected. These alpacas
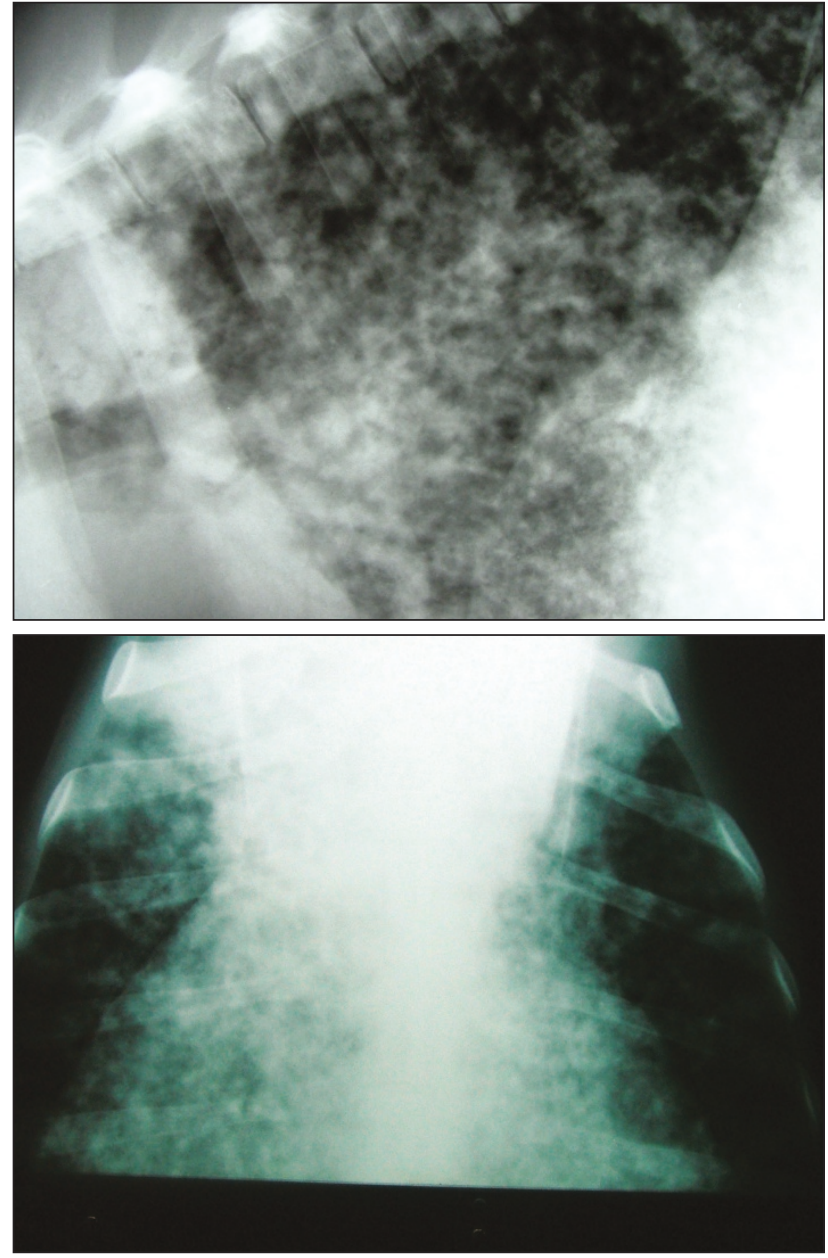

Figure 1a and b: A lateral (a) and ventrodorsal (b) thoracic radiograph from alpaca D prior to post mortem examination, highlighting severe, diffuse consolidation, suggestive of granulomatous, broncho-interstitial pneumonia.

were placed in isolation and further tests were conducted. Acid-fast bacteria could not be detected in cytospin smears following transtracheal lavage of both animals. The lymphocyte transformation assay (LTA) for TB (Dalley et al., 1999) was carried out on one ill animal (E) and found to be positive. Animal D died suddenly on November 21, 2004. The SICCT was carried out on E and the two remaining normal animals. However, SICCT testing was considered unsatisfactory due to the difficulty experienced in reading the test results. Therefore, testing was repeated using the SICAT test. All three alpacas tested negative.

\section{Post mortem examination}

On post mortem examination, animal D showed tuberculous lesions throughout the lungs and in the liver and hepatic, bronchial, mediastinal and mesenteric lymph nodes. Acid-fast bacteria typical of mycobacteria were detected in smears from these organs and in tracheal secretions. Disseminated miliary TB was noted. Following euthanasia of E, miliary TB was also confirmed on post mortem examination. Pulmonary involvement was less pronounced (that is, less lung tissue was affected), but abdominal lesions were more extensive. Granulomatouslike lesions were widespread within the peritoneum, mesentery and also present in the liver, kidneys, intestines 


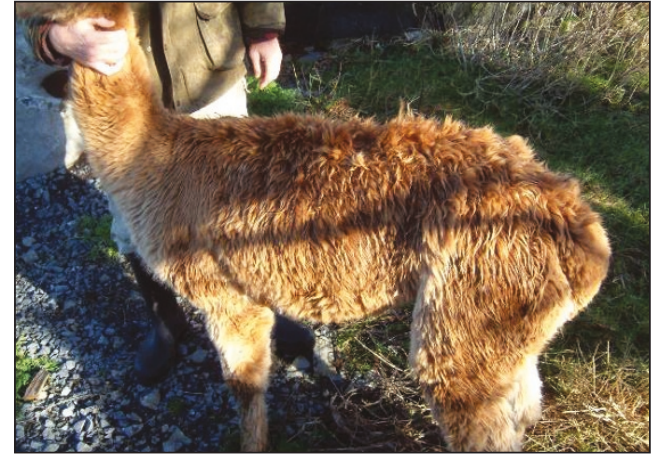

Figure 2: The low body condition score of alpaca $\mathrm{F}$ in

and mesenteric lymph nodes. Presumed infected, the two cohort animals were later euthanased but no gross pathological evidence of TB was detected.

\section{Bacteriology}

Bacteriology for M. bovis was carried out at the Central Veterinary Research Laboratory. Tissues from D and E were submitted for decontamination and culturing (Collins et al., 1997; Costello et al., 1998), and isolates were identified based on colony morphology, growth rate, pigmentation and cording characteristics (Collins et al., 1997) and by AccuProbe (Gen-Probe Inc., San Diego, California, USA) and GenoType MTBC (Hain Diagnostika, Nehren, Germany). M. bovis was isolated from tuberculous tissue from both animals.

\section{Subsequent investigation}

In the five months to April 2005, a further three ill alpacas died or were euthanased. Alpaca F (Figure 2) presented with mild respiratory symptoms on November 21, 2004, and was euthanased on January 10, 2005. Alpaca G died at pasture on February 15, 2005. Alpaca H presented on February 17, 2005 following progressive loss of weight over a 4-6 week period loss and was euthanased on April 19, 2005. Each of these cases was examined post mortem at the ARVL and had extensive miliary TB from which M. bovis was isolated. A composite post mortem examination report on all five cases indicated that lesions were present in the lung (Figure 3a, 3b and 3c), pleura, mesentery, spleen, liver, kidney, uterus, as well as bronchial mediastinal, mesenteric and prescapular lymph nodes (Figure 4). Tubercles were identified on histology (Figure 5), which typically had a caseous centre with foci of calcification, surrounded by lymphocytes and macrophages with epithelioid cells on the periphery. There was significant fibroblastic reaction, but giant cells were not seen. Isolates subsequently identified as $M$. bovis grew rapidly and luxuriantly on specific culture. In one case, the lesions in the lung indicated 'open TB', with caseo-necrotic lesions discharging into bronchioles.

\section{Discussion \\ General}

In many countries, TB in animals, generally due to infection with M. bovis, is of significant economic and zoonotic importance (O’Reilly and Daborn, 1995; Cousins and Dawson, 1999; Robert et al., 1999). Cases of TB in South American camelids (llama, alpaca, vicuña and guanaco) in their natural habitat are believed to be
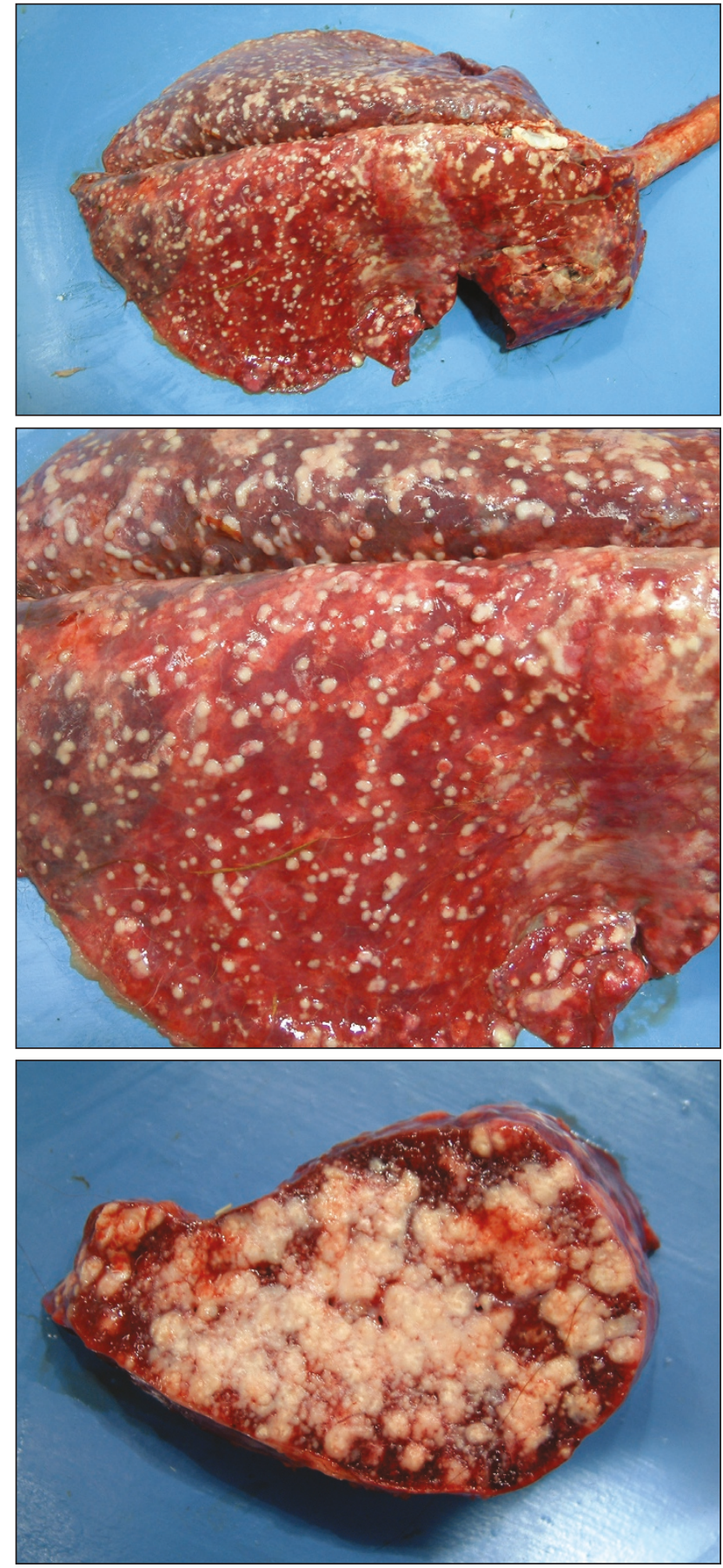

Figure 3a, b and c: Tuberculous lesions evident on gross post mortem examination on lung surface ( $a=$ top, $b=$ below) and on cross-section of lung tissue (c) (alpaca $\mathrm{D}$, November 2004).

rare, even though TB is endemic in local cattle, sheep and humans (Fowler, 1998). However, there are reports of infection in South American camelids held in close association with other infected animals, and grazing near infected cattle and badgers in the UK (Barlow et al., 1999; Twomey et al., 2007). TB in camelids is not due solely to $M$. bovis; M. microti (Oevermann et al., 2004) and M. kansasii (Johnson et al., 1993) have also been reported.

The predominant clinical sign seen in this herd was weight loss. Harsh lung sounds were also present, but only in advanced cases. There was no evidence or history of diarrhoea. At the initial farm visit, differential diagnoses 


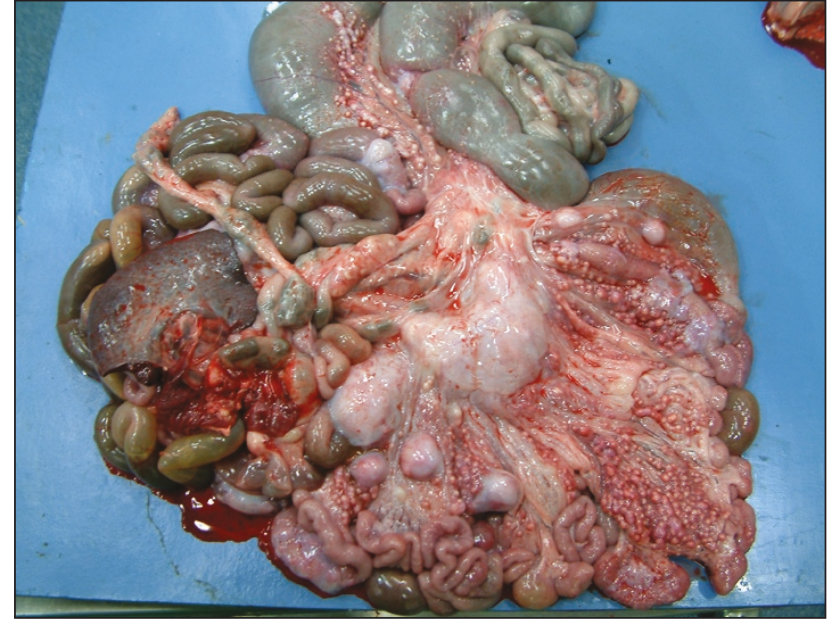

Figure 4: Tuberculous lesions evident on mesentery (alpaca D, November 2004).

were considered, including type 2 ostertagiosis, Johne's disease and hypocuprosis/trace element deficiencies (selenium/vitamin E), as ill-thrift and weight loss is a feature of each of these conditions, whereas diarrhoea is not common with ostertagiosis type 2 or hypocuprosis. Diarrhoea is a feature of Johne's disease in cattle, but not sheep (Radostits et al., 2007). There was no evidence of these conditions from the laboratory tests performed. TB has not been reported as a major health problem of camelids, either in their natural habitat in South America or when kept under farmed conditions in other countries. Regardless, Buick (2006) notes that TB should be considered in the differential diagnosis in all cases of ill-thrift in this species, with or without respiratory signs. In the outbreak reported here, clinical suspicion of $\mathrm{TB}$ was confirmed using post mortem examination, with confirmatory bacteriology and histopathology. Nonetheless, differential diagnoses will require investigation, especially if post mortem investigations cannot be carried out.

\section{Difficulty in the ante mortem diagnosis of $T B$}

The ante mortem diagnosis of TB in these cases was problematic. On this farm, the five cases were negative to all tuberculin tests. According to Buick (2006), the SICCT test offers reasonable sensitivity and specificity in alpaca, and is the official test in the UK for imported camelids. However, in the authors' experience, the SICCT (and particularly the intradermal injection of PPD) was difficult to perform consistently, due to the fine skin of the alpacas tested. It is for this reason that the authors also conducted the SICAT test (using the skin of the axilla rather than the cervical skin), as internationally advocated by some veterinary services and the llama/alpaca industry (Frost, 1999). Because each of these cases had advanced pathology, it is possible that they were anergic to these tests (Lepper et al., 1977). Although animal E was 'positive' to the LTA, the reaction was indicative of infection with $M$. avium rather than $M$. bovis, and it is worth noting that the LTA has not been validated for use in alpaca. According to Buick (2006), the IFN- $\gamma$ test $\left(\right.$ Bovigam $\left.^{\mathrm{R}}\right)$ is also not a valid test for the diagnosis of TB in camelids and therefore was not used. Furthermore, there is little evidence that

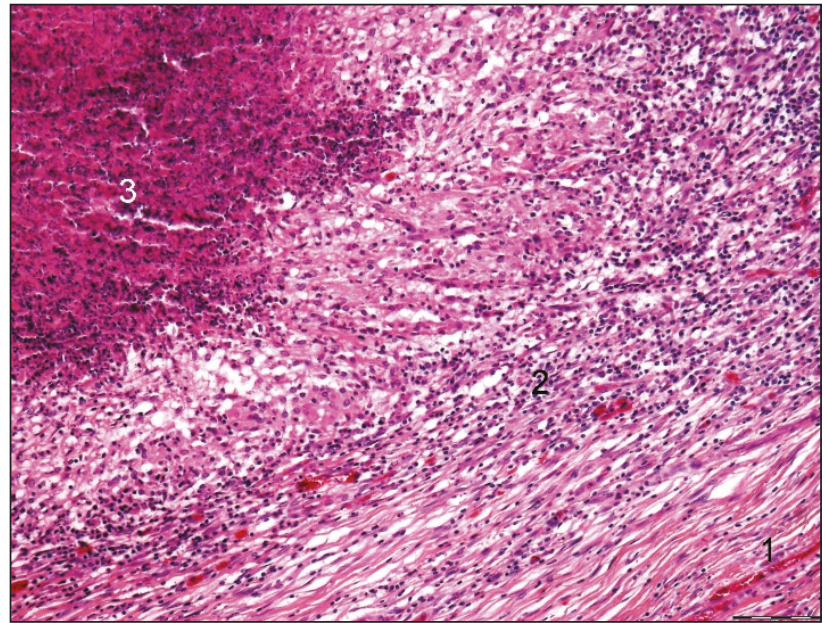

Figure 5: $\mathrm{H}$ and $\mathrm{E}$-stained section of lung tissue from alpaca $\mathrm{G}$ showing a granuloma with central caseous necrosis (3), surrounded by intense mononuclear cell reaction (2) and fibroblasts (1). (10x magnification.)

detection of humoral antibody (using methods such as the ELISA) would be a useful indicator of field infection (Cousins and Florisson, 2005). Among a broad range of other laboratory tests, only the A:G ratio proved useful. This finding is suggestive of a non-specific response to a long-term immune challenge, such as infection with $M$. bovis. In this herd, a low A:G ratio (specifically less than 1.0) was suggestive of infection risk. Indeed, the lower the A:G ratio, the higher the risk of disease being established. The absence of a reliable ante mortem test prohibited early identification of infected individuals.

\section{Post mortem examination}

Post mortem examination of alpacas was a significant part of the investigation permitting definitive diagnosis of TB heretofore presumptively diagnosed by clinical and clinicopathological means. Five alpaca showed well-established and extensive TB lesions confirmed by histopathology and bacteriology. The finding of so-called 'open TB', characterised by leakage of caseo-necrotic material into the bronchial tree, may have increased the risk of transmission of infectious aerosols to herd mates, to cattle in contiguous herds, to wildlife and to human contacts, with the alpaca herd acting as a propagating source. In the absence of a reliable ante mortem test, it was not possible to reliably identify individual alpaca as infected or diseased. Consequently, the whole flock had to be treated as one infected unit.

\section{Depopulation recommended}

The infection load within the herd was likely to have been high. Infection had established in at least five animals, and there may have been further infected animals prior to the on-farm investigation. The TB status of these latter animals was unknown as post mortem examinations had not been carried out. Against this, the two healthy cohorts sent to UVH with animals D and E, although presumed infected by contact, showed no gross lesions of TB on post mortem examination. It was also noted that the juvenile alpaca, which presented ill on November 8 , and which 
had shown weight loss along with a low A:G ratio, had recovered uneventfully. Despite these latter considerations, there appeared to be a significant risk of animal-to-animal and animal-to-human transmission within this herd. Primarily as a precautionary health and safety principle, total herd depopulation was recommended. However, this recommendation was rejected by the herd owners on financial grounds. In the absence of depopulation, protective measures were put in place, including isolation of diseased animals, euthanasia of cases with respiratory symptoms should they occur, improved animal hygiene measures and personal protective measures, e.g., using disposable gloves, masks, aprons, rubber boots, and disinfectant.

\section{Conclusion}

In retrospect, the diagnosis of TB in farmed alpaca in Ireland is not surprising. A range of risk factors are present, including: the presence of $M$. bovis in both South America and Ireland; the possible introduction of latent TB infection in imported alpacas; probable direct or indirect contact of alpacas with TB reactor cattle; and the uncontrolled movement of the badger, a TB reservoir species. However, at the time of diagnosis, a range of questions had not been answered, including the TB infection risk for alpaca farmed in areas where TB is endemic, the origin of the infection, the potential for alpaca-to-alpaca transmission and appropriate control measures, including the efficacy of predictor tests. These questions have been examined in a subsequent epidemiological investigation, and are presented separately (Connolly et al., 2008).

\section{Acknowledgement}

The authors thank Hester McAllister for expert radiological assistance.

\section{References}

Australian Alpaca Association Inc. (2001). Body condition score (BCS) of alpacas. Alpaca Note No 4/03, 2001. http://www.alpaca.org.nz/downloads/ bcs.pdf. Last accessed 11 January 2008.

Barlow, A.M., Mitchell, K.A. and Visram, K.H. (1999). Bovine tuberculosis in llama (Lama glama) in the UK. Veterinary Record. 145: 639-640. Buick, W. (2006). TB in domestic species other than cattle and badgers. Government Veterinary Journal 16: 87-91.

Connolly, D.J., More, S.J., Dwyer, P.J., Hayes, M., Ryan, E.G., Costello, E. and Kilroy, A. (2008). Tuberculosis in alpaca (Lama pacos) on a farm in Ireland. 2. Results of an epidemiological investigation. Irish Veterinary Journal 61 (8): 533-537.

Collins, C.H., Grange, J.M. and Yates, M.D. (1997). Tuberculosis

bacteriology: organization and practice. Second edition. Butterworth-

Heinemann, Oxford.

Costello, E., Quigley, F., Flynn, O., Gogarty, A., McGurk, J., Murphy,

A and Dolan, L. (1998). Laboratory examination of suspect tuberculosis lesions detected on abattoir post mortem examination of cattle from nonreactor herds. Irish Veterinary Journal 51: 248-250.

Cousins, D.V. and Dawson, D.J. (1999). Tuberculosis due to Mycobacterium bovis in the Australian population: cases recorded during 1970-1994.

International Journal of Tuberculosis and Lung Disease 3: 715-721.
Cousins, D.V. and Florisson, N. (2005). A review of tests available for use in the diagnosis of tuberculosis in non-bovine species. Revue scientifique et technique - Office International des Epizooties 24: 1039-1059.

Dalley, D., Chambers, M.A., Cockle, P., Pressling, W., Gavier-Widén, D. and Hewinson, R.G. (1999). A lymphocyte transformation assay for the detection of Mycobacterium bovis infection in the Eurasian Badger (Meles meles). Veterinary Immunology and Immunopathology 70: 85-94.

Fowler, M.E. (1998). Infectious diseases. In: Medicine and Surgery of South American Camelids (Llama, Alpaca, Vicuña, Guanaco). Second Edition. Iowa State University Press, Ames. pp 148-194.

Frost, B. (1999). Research update on diagnostic tests for tuberculosis in llamas/alpacas. Proceedings of the $102^{\text {nd }}$ Annual Meeting of the United States Animal Health Association, Minneapolis, Minnesota, USA. 3 - 9 October 1999.

Johnson, C.T., Winkler, C.E., Boughton, E. and Penfold, J.W.F. (1993).

Mycobacterium kansasii infection in a llama. Veterinary Record 133: 243-244.

Lepper, A.W.D., Pearson, C.W. and Corner, L.A. (1977). Anergy to

tuberculin in beef cattle. Australian Veterinary Journal 53: 214-216.

Oevermann, A., Pfyffer, G.E., Zanolari, P., Meylan, M. and Robert,

N. (2004). Generalized tuberculosis in llamas (Lama glama) due to

Mycobacterium microti. Journal of Clinical Microbiology 42: 1818-1821.

O'Reilly, L.M. and Daborn, C.J. (1995). The epidemiology of

Mycobacterium bovis infections in animals and man: a review. Tubercle and

Lung Disease 76 Supplement 1: 1-46.

Radostits, O.M., Gay, C.C., Hinchcliff, K.W. and Constable, P.D. (2007).

Veterinary Medicine: a textbook of the diseases of cattle, horses, sheep, pigs and goats. Saunders Elsevier, Edinburgh.

Robert, J., Boulahbal, F., Trystram, D., Truffot-Pernot, C., de Benoist

A-C., Vincent, V., Jarlier, V. and Grosset, J. (1999). A national survey of

human Mycobacterium bovis infection in France. International Journal of

Tuberculosis and Lung Disease 3: 711-714.

Twomey, D.F., Crawshaw, T.R., Anscombe, J.E., Farrant, L., Evans, L.J., McElligott, W.S., Higgins, R.J., Dean G., Vordermeier, M., Jahans, K. and de la Rua-Domenech, R. (2007). TB in llamas caused by Mycobacterium bovis. Veterinary Record 160: 170. 